\title{
An Interview with the Guest Editors
}

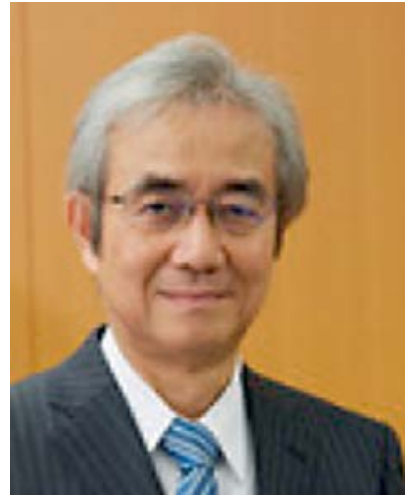

Dr. Hideki Kawanishi is Vice President and Director of the Department of Artificial Organs \& Surgery, Tsuchiya General Hospital and Clinical Professor at Hiroshima University of Medicine, Hiroshima, Japan. He has been an active clinical surgeon for the past 40 years and a nephrologist for 30 years and is a member of many national and international committees. He has published over 100 international articles in the fields of HD, PD, and artificial organs and currently holds editorial positions for three international journals.

At the symposium "Dialysis Situation in Asian Countries" held in February 2017, specialists from six economically developing countries joined specialists from Japan, an economically developed country, to discuss the current status of dialysis and the challenges remaining in the region. The articles in this publication report the contents of the discussions held.

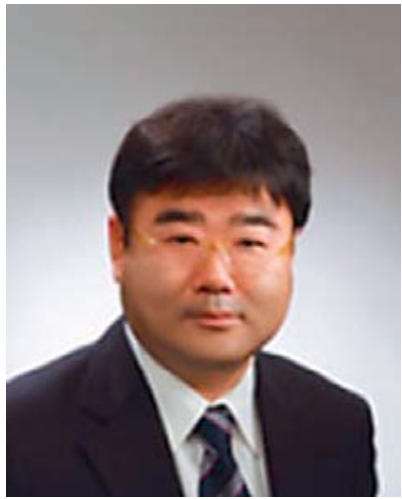

Dr. Toru Hyodo is currently Adjunct Lecturer in the Department of Urology, Kitasato University School of Medicine and Director of Eijin Clinic and Kurata Hospital Dialysis Center, Hiratsuka, Japan. His clinical and educational work extends outside Japan where, for example, he holds a guest professorship at Sen Sok International University Hospital, Cambodia, is Honorary President of both the Cambodian Association of Nephrology and the Ho Chi Min City Society of the Dialysis Therapy, Vietnam, and serves on the Oceania and South East Asia Regional Board of ISN.

Here, Guest Editors Dr. Hideki Kawanishi and Dr. Toru Hyodo answer some questions on the key points that were raised about dialysis in these initial discussions.

\section{KARGER}

E-Mail karger@karger.com

www.karger.com/bpu
(C) 2017 S. Karger AG, Basel 


\section{What have been the key developments in HD treatment across Asia in recent years?}

All economically developing countries in Asia now have access to many of the high-performance dialysis devices that have been developed and refined in economically advanced countries. Regardless of whether the individual devices were purchased by the country itself or were donated by another country, it is now possible across the region to use the same types of dialysis devices as those used in Japan. This does not necessarily mean that these devices are used in exactly the way that they are in Japan. Nevertheless, being equipped with the necessary devices to offer $\mathrm{HD}$ is a recent key development in the region.

\section{What have been the driving forces behind these key developments?}

Today, due to advances in internet technologies, physicians in economically developing countries can learn in real time about the latest treatments provided in advanced countries. Moreover, due to economic improvements, they can attend and acquire information at meetings held by the American Society of Nephrology (ASN), European Dialysis and Transplant Association, World Congress of Nephrology (WCN), and Japanese Society for Dialysis Therapy (JSDT).

A good example of a key development happening under such circumstances is the introduction of online hemodiafiltration (HDF) in Vietnam. Back in 2011, postdilution online HDF started being provided at Bach Mai Hospital in Hanoi. HDF was subsequently introduced in Lao PDR and Myanmar in 2016. Needless to say, economic development is always the driving force behind key developments in the region.

\section{Among the many challenges in HD provision faced by economically developing countries in Asia, as summarized in the figures for each country in Toshiaki Masuda's opening article of this special issue, what do you see as the top 5 challenges in the region?}

We see the top issue as the harsh reality of "no money, no life" among patients with end-stage renal disease (ESRD). With no national health insurance system in place in Cambodia or Lao PDR, patients are responsible for covering the cost of dialysis, with one dialysis session costing about $30-50 \%$ of the average monthly wage there. This means dialysis is a privilege of the wealthy. In India and Myanmar, although financial support is provided to dialysis patients, it is not enough to cover all of the expenses associated with dialysis. In the case of Vietnam and Mongolia, dialysis is covered by a national health insurance system, but the financial burden of dialysis for patients is still large.

As a second challenge, issues associated with a country's national health insurance system are closely linked to its economic development. Therefore, all we can do is to wait and see what changes occur in the future.

Third, because many HD centers are located in metropolitan areas, patients living in rural areas need to travel to big cities for dialysis. In Cambodia especially, where most dialysis centers are concentrated in Phnom Penh and Siem Reap, the cost of dialysis includes expenses for transportation, accommodation, dialysis itself, and medication such as erythropoietin, iron supplements, and antihypertensives. As a result, physicians specialized in dialysis also frequently face this reality of "no money, no life." In this situation, continuous ambulatory peritoneal dialysis (CAPD) is advantageous. However, in reality, the international manufacturers of peritoneal dialysis systems and supplies who have to contend with underdeveloped transportation systems, such as in Cambodia, Myanmar, and Lao PDR, are hesitant to export and commercialize CAPD systems.

A fourth challenge is that all economically developing countries in Asia are behind in keeping dialysate clean. This is largely because most do not have a job category equivalent to clinical engineering (which requires professional knowledge and skills for the setting up and maintenance of medical devices). Because of this, the knowledge and skills needed to purify dialysate are lacking, and daily maintenance of dialysis devices is not adequately provided.

Lastly, it is important for economically developing countries in Asia to establish academic societies like the Japanese Society of Nephrology and JSDT. Academic societies can receive support from the International Society of Nephrology (ISN) to make it easier for economically developed countries to share dialysis know-how with economically developing countries. The Society of Dialysis Therapy of Ho Chi Minh City was established in 2015 for physicians, nurses, and other healthcare professionals across Vietnam. The Cambodian Association of Nephrology was established in 2016 and was approved as an ISNaffiliated society in 2017. And Lao PDR plans to establish a Society of Nephrology within 2017.
20

Blood Purif 2017;44(suppl 1):19-21 DOI: $10.1159 / 000479578$
Kawanishi/Hyodo 


\section{Japan is among the economically developed countries offering support to advance HD in economically developing countries in Asia. What have been the key components of that support so far and will they remain the same for the foreseeable future?}

As part of Japan's support activities, we have been conducting water quality surveys in Vietnam, Cambodia, and Myanmar since 2011 (see the article by Dr. Kenichi Kokubo and coauthors in this special issue for details, pp. 5561). The survey data confirmed the need for disseminating knowledge and skills in dialysate purification as well as for quickly incorporating endotoxin retentive filters into the dialysis console. Endotoxin retentive filters are currently being incorporated into dialysis consoles in Lao PDR, Cambodia, Myanmar, and Mongolia by a collaborative team of members from NGO Ubiquitous Blood Purification International (UBPI), the Japanese Society for Technology of Blood Purification, and the Japan Association for Clinical Engineers, in cooperation with Japanese manufacturers such as Nipro Corporation.

Also, in 2017, through the efforts of NGO UBPI and a collaborative team working as an intermediary, the governments of Myanmar and Japan decided to establish a clinical engineering course at a university in Myanmar.
Because NGO UBPI members belong to various academic societies, it gives NGO UBPI a special feature - it allows us to inform the world about the challenges associated with dialysis. We do this by presenting survey data at society meetings (ASN, JSDT, WCN, etc.) and in Englishlanguage journals (Nephron Extra, Renal Replacement Therapy, etc.). We believe this approach is effective and plan to continue it into the future.

\section{How do you see further developments in dialysis moving forward in the region? What are the next steps to consider?}

First of all, the incorporation of CAPD is an urgent matter, especially in Cambodia, Lao PDR, and Myanmar. CAPD is essential for treating patients with ESRD in rural districts.

Second, we are hastening to establish academic societies in these countries. We think our next step is to establish a system that helps distribute ISN's educational support throughout Asia - by developing a close collaborative relationship with the society and by attending ISN meetings and using ISN's online educational systems. We believe this will enable us to treat as well as prevent ESRD in the region. 